\title{
Reflection of Adjuvant Treatment Approaches for Early Stage Testis Tumors in Our Clinic
}

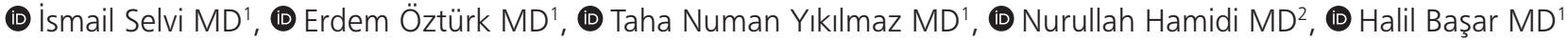 \\ 'University of Health Sciences, Dr. Abdurrahman Yurtaslan Ankara Oncology Training and Research Hospital, Clinic of Urology, Ankara, Turkey \\ ${ }^{2}$ Ankara Atatürk Training and Research Hospital, Clinic of Urology, Ankara, Turkey
}

\begin{abstract}
Objective: Treatment modalities applied after orchiectomy in early-stage germ cell tumors (GCTs) include significant changes in each new study. In this study we reevaluated the treatment approaches used in our hospital between 2010-2014 according to current guidelines.

Materials and Methods: We retrospectively evaluated the oncologic treatments and follow-up data of 32 patients who underwent radical orchiectomy between January 2010 and December 2014 due to testicular tumor and were diagnosed with early stage GCT in the Urology Clinic of Dr. Abdurrahman Yurtaslan Ankara Oncology Training and Research Hospital of University of Health Sciences.

Results: Of 19 patients diagnosed with stage 1 seminomas, 3 patients in the low risk group were followed. Of 4 patients who received single-dose carboplatin therapy, 2 were at low risk and 2 were at high risk. Therefore, 2 patients at low-risk had overtreatment. Twelve patients were treated with radiotherapy (RT) that was no longer recommended in guidelines after 2014. Two patients in the low risk group of stage 1 non-seminoma were followed. One of them had recurrence at 12 months, and received 3 cycles of bleomycin + etoposide + cisplatin (BEP) according to current guidelines. Four patients with stage 1 non-seminoma underwent 2 cycles of BEP because they were considered at high risk. These patients are now recommended to receive 1 cycle BEP according to the current guidelines. While 4 patients with stage 1 mixed GCT were followed because of low risk, one patient was administered 2 cycle of BEP based on the old guidelines, at that time because of high risk. In the seminoma group that was administered RT, acute myeloblastic leukemia and oligospermia toxicity were detected, but these were not observed in the carboplatin group. One of high-risk non-seminoma patients who received 2 doses of BEP developed Myelodysplastic syndrome.

Conclusion: Early-stage GCTs have high cancer-specific and overall survival rates with appropriate treatment approaches. Although there are still controversial issues regarding their management, treatment approaches are changing with each study. Therefore, it is crucial to remain informed about current international guidelines and new scientific studies.
\end{abstract}

Keywords: Adjuvant treatments, early stage, current guidelines, radical orchiectomy, testicular tumor

\section{Introduction}

Testicular cancer accounts for $1-1.5 \%$ of all cancers in men and $5 \%$ of male urological malignancies. It is usually seen between the ages of 15 and 35 . In the USA, it is the most common malignancy among men aged 20-40 years and the second most common malignancy after leukemia among adolescents aged 15-19 years. The prevalence of bilateral germ cell tumor (GCT) is
$2.5 \%$, with $0.6 \%$ of these being synchronous tumors and $1.9 \%$ being metachronous contralateral tumors (1).

The vast majority (90-95\%) of testicular tumors are GCTs. The most common histological type of GCT is seminoma (55\%), which peaks between the ages of 30-40 years. Non-seminomas account for $40 \%$ and peak between the ages of $20-30$ years (2). Stage 1 seminomas comprise the majority (80-85\%) of GCTs diagnosed in daily practice $(3,4)$. The 10 -year overall

Address for Correspondence: İsmail Selvi MD, University of Health Sciences, Dr. Abdurrahman Yurtaslan Ankara Oncology Training and Research Hospital, Clinic of Urology, Ankara, Turkey Phone: +90 3123360909 E-mail: ismselvi33@hotmail.com ORCID-ID: orcid.org/0000-0003-3578-0732 Received: 05.01.2018 Accepted: 10.02.2018 
survival (OS) rates for stage 1, which is considered early stage, are over $95 \%$.

The primary treatment is inguinal orchiectomy, and postoperative treatment methods vary depending on the tumor's stage and histopathological type. Postoperative treatment options include long-term active surveillance, radiotherapy (RT), and chemotherapy (CT) for stage 1 seminomas and CT, retroperitoneal lymph node dissection (RPLND), and active surveillance for stage 1 non-seminomas; however, the strength of these recommendations has changed continuously (1).

In this study, we aimed to present the treatment approaches used in our clinic after radical orchiectomy for seminomas and non-seminomas in patients with early testicular cancer and to discuss these in light of the updated 2017 guidelines of the European Association of Urology (EAU).

\section{Materials and Methods}

We retrospectively reviewed the pathology and radiological imaging results of 77 patients who underwent radical orchiectomy due to testicular tumor in the Urology Clinic of Dr. Abdurrahman Yurtaslan Ankara Oncology Training and Research Hospital of University of Health Sciences between January 2010 and December 2014. Because the study was a retrospective data analysis, ethics committee approval was not sought. The patients were given information about the study and informed postoperatively that their oncological data such as the recurrence, and overall survival would be evaluated without using their names. Of the 77 patients screened, we included the oncological treatment and follow-up data of 32 patients who had early testicular tumors (stage 1 ) according to clinical staging. The stage 1 patients were evaluated in terms of age at diagnosis, tumor histopathology, postoperative follow- up, RT and CT received, occurrence of relapse during follow-up, treatment received for relapse, mean follow-up period, diseasefree survival (DFS), and OS. We aimed to evaluate changes in adjuvant therapy approaches by comparing the treatments administered to each patient during the study period to the adjuvant therapy currently recommended according to the riskbased treatment options in the current EAU guidelines.

\section{Results}

GCTs were detected in 69 of the 77 patients who underwent inguinal orchiectomy for a diagnosis of testicular tumor. Of these patients, 32 were stage 1, 4 were stage IS, 9 were stage 2 , and 24 were stage 3 . The 32 patients who had stage 1 testicular cancer were included in the study.

The mean age of these patients was $32.6 \pm 10.61$ (8-60) years. Nineteen patients (54.9\%) had pure seminoma (17 classical, 1 anaplastic, 1 spermatocytic), 7 patients (21.8\%) had nonseminoma tumors, and 6 patients $(18.8 \%)$ had mixed GCTs. Two patients $(6.25 \%)$ had bilateral synchronous stage 1 seminomas. Tumors were located on the left side in 10 patients (31.2\%), on the right side in 20 patients $(62.5 \%)$, and bilaterally in 2 patients (6.25\%). The distribution of the patients based on their histopathological features is presented in Table 1. Mean tumor size was $4.48 \pm 2.04(1.2-9) \mathrm{cm}$. Intratubular germ cell neoplasia was detected in 17 patients (40.47\%).

Nine patients (28.12\%), including 3 with seminoma, 2 with non-seminoma, and 4 with mixed GCTs were scheduled for follow-up. Of these 9 patients, 1 patient with a seminoma and 1 patient with a mixed GCT did not attend follow-up regularly. Recurrence was detected at 12 months in 1 patient with non-seminoma. This patient underwent $C T$ with 3 cycles of bleomycin + etoposide + cisplatin (BEP) protocol and exhibited

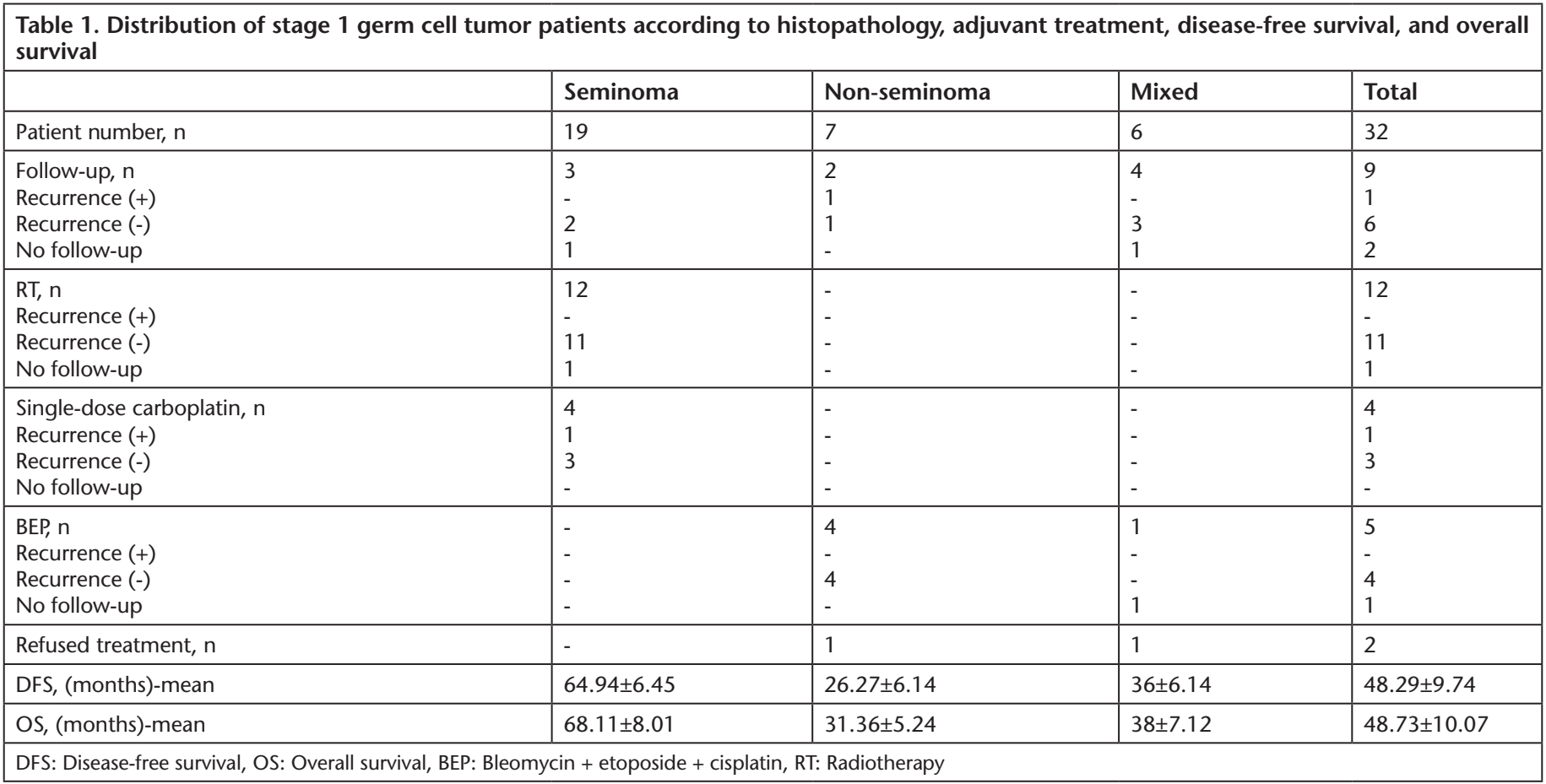


no recurrence in the following 12-month follow-up period. The mean follow-up period of the other 6 patients who were followed was $40 \pm 10.7$ (32-64) months and no recurrence was observed.

Twelve patients with seminoma (37.5\%) were treated with RT. Although 1 of these patients was lost to follow-up after RT, the other 11 exhibited no recurrence during the mean followup period of $64 \pm 11.8$ (28-76) months. Five of the 12 patients underwent RT of $20 \mathrm{~Gy}$ in 10 fractions to the lymph nodes in the paraaortic region and ipsilateral pelvis (hockey stick field), while the other 7 patients were treated with RT of 20 Gy applied unilaterally to the paraaortic region. Of the 11 patients followed after RT, 1 patient developed acute myeloblastic leukemia (AML) thought to be secondary to RT at 57 months. Oligospermia developed postoperatively in 2 patients and showed no improvement even at 70 months after RT.

As adjuvant CT, 4 patients (12.5\%) received a single dose of carboplatin because they had stage 1 seminomas, whereas 5 patients (15.6\%) with non-seminomas and mixed GCTs received BEP. One of the patients who received a single dose of carboplatin had bilateral seminomas, developed recurrence at 23 months, and subsequently underwent 4 cycles of BEP. One of the 5 BEP-treated patients was not followed after CT, but no recurrence was observed in the other 4 patients during a mean follow-up period of $52 \pm 23.12$ (18-76) months. Only 1 patient (25\%) developed Myelodysplastic syndrome (MDS) at 72 months.

With the exception of the patient who developed AML and 2 patients with persistent oligospermia, no acute or late severe (grade $>2$ ) toxicity was observed in any of the patients in the other groups. Mean DFS of the patients was 48.29 \pm 9.74 (2160) months and OS was 48.73 \pm 10.07 (24-64) months (Table 1).

Table 2 shows the comparison of the adjuvant therapies provided to these patients at our clinic between January 2010 and December 2014, as determined in our retrospective analysis, and the adjuvant therapies recommended in the EAU 2017 updated guidelines.

\section{Discussion}

Seminomas have an occult metastasis rate of $10-15 \%$ and systemic recurrence rate of $1-4 \%$ after retroperitoneal therapy (5). Poor prognostic factors for relapse in seminoma are a primary tumor size larger than $4 \mathrm{~cm}$ and the presence of rete testis invasion.

Twenty years ago, RT administered as a dog-leg field to the retroperitoneal and ipsilateral pelvic lymph nodes as adjuvant therapy for stage 1 seminomas had a success rate of $95 \%$ (6), with post-RT relapse rate $<5-10 \%$. Disease-specific survival reached $100 \%$ with BEP administered at relapse (7). In later years, evidence showing that single-dose carboplatin provided similar oncological outcomes with fewer side effects led to RT losing favor as a first-line treatment (8).

Since 2014, the EAU guidelines have no longer recommended adjuvant RT for stage 1 seminomas due to the long-term risks of RT in terms of cardiovascular toxicity (9.6\% of patients within 5-8 years), gonadal toxicity ( $8 \%$ permanent oligospermia), and secondary malignant neoplasm (18\% risk in 25 years, most commonly leukemia) (9).

In the present study, treatment planned before 2014 for the 12 seminoma patients included RT of 20 Gy delivered in 10 fractions in a hockey-stick field to the paraaortic and ipsilateral pelvic lymph nodes in 5 patients, and 20 Gy RT applied unilaterally to the paraaortic area in 7 patients. If treatment were planned for these 12 patients today, none would undergo RT (Table 2).

Single-dose carboplatin began to appear in guidelines as an A-level recommendation in 2010 because it provided the same oncological efficacy as RT with fewer side effects (10). As of 2014, the EAU guidelines prioritized active surveillance for stage 1 seminoma and recommended single-dose carboplatin in the presence of risk factors (9). According to Ondrusova et al. (11), recurrence is detected in the retroperitoneum after adjuvant single-dose carboplatin in $9.1 \%$ of patients, but that a cure can be achieved in these cases with 3 doses of BEP. Chau et al.

\begin{tabular}{|c|c|c|}
\hline Stage 1 GCT groups & Adjuvant treatment given & $\begin{array}{l}\text { Adjuvant treatment recommended } \\
\text { in EAU } 2017 \text { guideline }\end{array}$ \\
\hline High-risk seminoma & $\begin{array}{l}\text { Unilateral RT to the paraaortic area }(n=1) \\
\text { RT in hockey-stick field (unilateral paraaortic area + } \\
\text { ipsilateral pelvic bone) }(n=5) \\
\text { Single-dose carboplatin }(n=2)\end{array}$ & $\begin{array}{l}\text { Single-dose carboplatin }(n=1) \\
\text { Single-dose carboplatin }(n=5) \\
\text { Single-dose carboplatin }(n=2)\end{array}$ \\
\hline Low-risk non-seminoma & Follow-up $(n=2)$ & Follow-up $(n=2)$ \\
\hline Recurrence of low-risk non-seminoma during follow-up & 3 cycles of BEP $(n=1)$ & 3 cycles of BEP $(n=1)$ \\
\hline High-risk non-seminoma & 2 cycles of BEP $(n=4)$ & 1 cycle of BEP $(n=4)$ \\
\hline
\end{tabular}


(12) claimed that the timing of CT does not affect oncological outcomes, but in practice they recommend initiating $\mathrm{CT}$ within the first 60 days after orchiectomy.

In our study, single-dose carboplatin was administered to 4 patients with stage 1 seminomas. Two of these patients were low-risk, and active surveillance would have been more appropriate as an initial management strategy according to current guidelines (Table 2).

The growing trend toward active surveillance stems from the fact that patients under active surveillance have a relapse rate of $15-20 \%$ and these cases can be treated effectively with RT or CT. The average time to relapse during active surveillance is 12-18 months, though $29 \%$ of relapses occur after longer intervals (13). In light of these data, active surveillance was included as a level B recommendation in guidelines starting in 2010 (10). Since 2014, the EAU guidelines endorse active surveillance as a level A recommendation for stage 1 seminoma (9). The effect of these risk factors (rete testis invasion and tumor size) on oncological outcomes is still debated $(14,15,16,17)$.

In our study, only 3 of the 19 stage 1 seminoma cases was followed with active surveillance. However, based on current knowledge, this approach could have been applied with 8 other low-risk patients. It can be concluded that single-dose carboplatin in 2 and RT in 6 of those 8 patients was unnecessary (Table 2).

In the literature, secondary malignancies (predominantly leukemia, as well as renal, lung, and pancreatic cancers and neurofibrosarcoma) are reported at a rate of $5-8 \%$ in patients with stage 1 seminomas who have undergone RT (18). Similarly, in our study, AML was observed in 1 patient (9\%) after RT, while no secondary malignancy was observed in the carboplatin or active surveillance groups. While it is reported that fertility rates improve 5 years after adjuvant RT and that $8 \%$ of patients have permanent oligospermia, we found that 2 patients (18\%) in our study experienced persistent oligospermia even after 70 months as late toxicity. This toxicity was not observed in the carboplatin and active surveillance groups.

While one-third of non-seminomas are diagnosed at stage 1 , more than $30 \%$ of these have occult metastasis at time of diagnosis and $30 \%$ will recur without additional treatment. The most important prognostic indicator for occult metastasis in this group is the presence of lymphovascular invasion (LVI). Approximately $33 \%$ of all patients with stage 1 non-seminomas have LVI (19). Other important prognostic risk factors are a proliferation rate $>70 \%$ and percentage of embryonal carcinoma $>50 \%$. In the presence of LVI, relapse occurred in $14-22 \%$ and metastasis developed in $48 \%$ of patients who did not receive postoperative adjuvant therapy (20).

The 20-year long-term relapse rate of patients under active surveillance is $27-30 \%$ (21). Eighty percent of these relapses occur within the first 12 months, $12 \%$ occur in the second year, $6 \%$ occur in the third year, and the rate falls to $1 \%$ in the $4^{\text {th }}$ and $5^{\text {th }}$ years (21). In this group, 5 years of close follow-up is imperative as routine practice (22). Computed tomography done at 3 and 12 months is very valuable. It has been determined that computed tomography using a low-dose protocol, which reduces the risk of secondary malignancy due to radiation exposure through frequent computed tomography scans, reduces the risk of radiation by 55\% (23).

In our study, 2 low-risk patients from the 7 patients with stage 1 non-seminomas were managed with active surveillance. Only 1 patient had relapse at 12 months and received 3 cycles of BEP. Although this treatment approach was implemented before 2014, it still complies with current guidelines (Table 2).

Two-dose BEP CT has been recommended for many years to reduce the risk of relapse in the presence of LVI. In a Swedish and Norwegian Testicular Cancer Group (SWENOTECA) study carried out in 2009, it was shown that single-dose BEP therapy in the presence of LVI reduced relapse to $2.3 \%$ over 8.1 years of follow-up and caused fewer side effects (14), and BEP was subsequently included as a level A recommendation in EAU guidelines as of 2014. Three or 4 doses of BEP is recommended for relapses occurring after single-dose BEP (24).

These patients may experience pulmonary toxicity (pneumonitis, pulmonary fibrosis, etc.) due to bleomycin, which can be fatal in $1-3 \%$ of patients (25). There may be a 3.1-fold higher risk of cardiovascular disease (myocardial infarction, coronary artery disease, etc.) compared to the normal population after BEP administration (26). Although Chamie et al. (27) reported higher risk of secondary solid malignancy due to CT some studies report a $0.5-1 \%$ risk of hematological malignancy as a result of high-dose etoposide (28). The adverse effect of cisplatin-based CT on fertility and sexual function is not yet known (29).

In our study, 4 patients with stage 1 non-seminomas were treated with 2 doses of BEP because they were considered high risk. These patients were treated before the latest changes in the guidelines. Had they been diagnosed today, they would be candidates for a single-dose BEP protocol (Table 2). In only 1 of these 4 patients (25\%), MDS appeared as a secondary hematologic malignancy at 72 months. This rate seems to be high due to the low number of patients and short follow-up times, and since there was no group in our study that received single-dose BEP, we were unable to compare the relative effect of receiving 1 and 2 doses of BEP.

In a 2008 study by the German Testicular Cancer Study Group, the 2-year DFS rate was $99.41 \%$ in the group that received single-dose BEP and $92.37 \%$ in the RPLND group (30). Therefore, although RPLND is the most common treatment for stage 1 non-seminoma in the USA, is recommended as first-line treatment for high-risk patients in current EAU guidelines, and it is less effective than CT with single-dose BEP. Similar to the EAU guidelines, none of the 7 patients with stage 1 seminomas underwent RPLND in our study (Table 2).

Due to their non-seminoma component, mixed GCTs are also treated like non-seminomas. Four of the 6 patients with stage 1 mixed GCTs in our study were followed because they were considered low-risk, while 1 high-risk patient received 2 cycles of BEP based on previous guidelines (Table 2).

The other patient refused treatment and was not followed (Table 1). For non-seminomas and GCTs with vascular invasion (VI), single-dose BEP is the first-line treatment, and follow-up or RPLND are alternative options. In the absence of VI, follow-up is preferred, and single-dose BEP or RPLND are recommended as alternative options. 
In the field of urooncology, new research can lead to drastic and frequent changes, especially in post-orchiectomy treatment approaches for stage 1 testicular tumors. This is clearly demonstrated by the fact that most treatment approaches implemented correctly with the patients in our study according to the guidelines of seven years ago are now obsolete. Our results support the prevailing view that carboplatin for stage 1 seminomas is safer than RT in terms of late cardiovascular toxicity, gonadal toxicity, and secondary malignant neoplasm; however, the results of long-term follow-up are needed to confirm this.

\section{Study Limitations}

The limitations of study include short follow-up periods, a small patient population, and the retrospective design of the study. The new treatment schemes presented in the EAU guidelines after 2014 indicate similar cancer survival rates with fewer side effects. However, prospective studies involving larger numbers of patients followed for longer periods will provide a more realistic view of the effects of the existing guidelines on toxicity and patient survival.

\section{Conclusion}

With current treatment approaches, testicular tumors detected in the early stage have a high likelihood of being cured and show low relapse rates. Although postoperative follow-up and adjuvant therapy options vary depending on the tumors' histopathology and risk factors, OS rates are quite high. Due to these excellent survival rates and the possible side effects of adjuvant therapy, less toxic treatment options are also preferred in the current EAU guidelines, and treatment protocols can change nearly every year. Therefore, it is essential in clinical practice that every center that deals with testicular tumors stays informed about emerging studies and guidelines.

\section{Ethics}

Ethics Committee Approval: Ethics committee approval was not sought for this retrospective study.

Informed Consent: The patients were given information about the study and informed postoperatively that their oncological data such as the recurrence, and overall survival would be evaluated without using their names.

Peer-review: Externally and internally peer-reviewed.

\section{Authorship Contributions}

Surgical and Medical Practices: H.B., Concept: T.N.Y., N.H., Design: E.Ö., T.N.Y., Data Collection or Processing: I.S., Analysis or Interpretation: E.Ö., Literature Search: I.S., Writing: I.S.

Conflict of Interest: No conflict of interest was declared by the authors.

Financial Disclosure: The authors declared that this study received no financial support.

\section{References}

1. Stephenson AJ, Gilligan TD. Neoplasms of the Testis. In: CampbellWalsh Urology 11th Edition. Philadelphia: Elsevier Press, 2016;784-814.
2. Ruf CG, Isbarn H, Wagner W, et al. Changes in epidemiologic features of testicular germ cell cancer: age at diagnosis and relative frequency of seminoma are constantly and significantly increasing. Urol Oncol 2014;32:33.

3. Sokoloff MH, Joyce GF, Wise M. Urologic Diseases in America Project: Testis cancer. J Urol 2007;177:2030-2041.

4. Heinzelbecker J, Katzmarzik M, Weiss C, et al. Changes of stage, predictive factors and adjuvant treatment modalities in seminomatous testicular cancer from 1987 to 2007 and their impact on the status of metastasis, recurrence-free and overall survival: a single-center analysis. Urol Int 2011;87:282-287.

5. Alan J Wein, Kavoussi LR, Novick AC, et al. Neoplasm of the Testis. In: Campbell-Walsh Urology. 10th Edition. Philadelphia: Saunder Elseiver, 2012;837-870.

6. Kuzgunbay B. The Treatment Modalities in Stage 1 Seminoma; Alterations in Last Five Years. Bulletin of Urooncology 2015;14:228-231.

7. Chung $P$, Warde P. Surveillance in stage 1 seminoma. Urol Oncol 2006;24:75-79.

8. Dieckmann KP, Dralle-Filiz I, Heinzelbecker J, et al. Seminoma Clinical Stage 1 - Patterns of Care in Germany. Urol Int 2016;96:390-398.

9. Albers P, Albrecht W, Algaba F, et al. Guidelines on testicular cancer. Eur Urology 2014;1:1-54.

10. Albers P, Albrecht W, Algaba F, et al. Guidelines on testicular cancer. Eur Urology 2010;1:1-51.

11. Ondrusova M, Ondrus D, Miskovska V, et al. Management of clinical stage I testicular seminoma: active surveillance versus adjuvant chemotherapy. Int Urol Nephrol 2015;47:1143-1147.

12. Chau C, Cathomas R, Wheater M, et al. Treatment outcome and paterns of relapse following adjuvant carboplatn for stage I testcular seminomatous germ cell tumour: results from a 17 year UK experience. Ann Oncol 2015;26:1865-1870.

13. Albers $P$, Albrecht W, Algaba F, et al. Guidelines on testcular cancer: 2015. Update. Eur Urol 2015;68:1054-1068.

14. Cohn-Cedermark G, Stahl O, Tandstad T; SWENOTECA. Surveillance vs. adjuvant therapy of clinical stage I testcular tumors - a review and the SWENOTECA experience. Andrology 2015;3:102-110.

15. Mortensen MS, Lauritsen J, Gundgaard MG, et al. A natonwide cohort study of stage I seminoma patents followed on a surveillance program. Eur Urol 2014;66:1172-1178.

16. Ruf CG, Khalili-Harbi N, Sachs $S$, et al. The search for biomarkers of metastatic seminoma. J Urol 2013;190:1046-1051.

17. Dieckmann KP, Dralle-Filiz I, Matthies C, et al. Testicular seminoma clinical stage 1: treatment outcome on a routine care level. J Cancer Res Clin Oncol 2016;142:1599-1607.

18. De Felice F, Musio D, Gravina GL, et al. Adjuvant radiation therapy in stage I seminoma: 20 years of oncologic results. Oncotarget 2016;7:80077-80082.

19. Tandstad T, Ståhl O, Håkansson U, et al. One course of adjuvant BEP in clinical stage I nonseminoma mature and expanded results from the SWENOTECA group. Ann Oncol 2014;25:2167-2172.

20. Read G, Stenning SP, Cullen MH, et al. Medical Research Council prospective study of surveillance for stage I testicular teratoma. Medical Research Council Testicular Tumors Working Party. J Clin Oncol 1992;10:1762-1768.

21. Oliver RT, Ong J, Shamash J, et al. Long-term follow-up of Anglian Germ Cell Cancer Group surveillance versus patients with Stage 1 nonseminoma treated with adjuvant chemotherapy. Urology 2004;63:556-561.

22. Vidal AD, Thalmann GN, Karamitopoulou-Diamantis $E$, et al. Long-term outcome of patients with clinical stage I high-risk nonseminomatous germ-cell tumors 15 years after one adjuvant cycle of bleomycin, etoposide, and cisplatin chemotherapy. Ann Oncol 2015;26:374-377. 
Selvi et al.

Adjuvant Treatment Approaches for Early Stage Testis Tumor

23. O'Malley ME, Chung P, Haider M, et al. Comparison of low dose with Standard dose abdominal/pelvic multidetector CT in patients with stage 1 testicular cancer under surveillance. Eur Radiol 2010;20:1624-1630.

24. Albers P, Albrecht W, Algaba F, et al. Guidelines on testicular cancer. Eur Urology 2017;1:1-54.

25. Haugnes HS, Bosl G], Boer $\mathrm{H}$, et al. Long-term and late effects of germ cell testicular cancer treatment and implications for follow-up. J Clin Oncol 2012;30:3752-3763.

26. Haugnes HS, Wethal T, Aass N, et al. Cardiovascular risk factors and morbidity in long-term survivors of testicular cancer: a 20-year follow-up study. J Clin Oncol 2010;28:4649-4657.

27. Chamie K, Kurzrock EA, Evans CP, et al. Secondary malignancies among nonseminomatous germ cell tumor cancer survivors. Cancer 2011;117:4219-4230.

28. Westermann DH, Studer UE. High-risk clinical stage I nonseminomatous germ cell tumors: the case for chemotherapy. World J Urol 2009;27:455-461.

29. Böhlen D, Burkhard FC, Mills R, et al. Fertility and sexual function following orchidectomy and 2 cycles of chemotherapy for stage I high risk nonseminomatous germ cell cancer. J Urol 2001;165:441-444.

30. Albers P, Siener R, Krege S, et al. Randomized phase III trial comparing retroperitoneal lymph node dissection with one course of bleomycin and etoposide plus cisplatin chemotherapy in the adjuvant treatment of clinical stage I Nonseminomatous testicular germ cell tumors: AUO trial AH 01/94 by the German Testicular Cancer Study Group. J Clin Oncol 2008;26:2966-2972. 